\title{
Structural analysis of dextrins and characterization of dextrin-based biomedical hydrogels
}

\author{
Dina M. Silva ${ }^{\mathrm{a}, 1}$, Cláudia Nunes ${ }^{\mathrm{b}, 1}$, Isabel Pereira ${ }^{\mathrm{a}}$, Ana S.P. Moreira ${ }^{\mathrm{b}}$, \\ Maria Rosário M. Domingues ${ }^{\mathrm{b}}$, Manuel A. Coimbra ${ }^{\mathrm{b}}$, Francisco M. Gama ${ }^{\mathrm{a}, *}$ \\ ${ }^{a}$ CEB-Centre of Biological Engineering, IBB-Institute for Biotechnology and Bioengineering, Department of Biological Engineering, University of Minho, \\ Campus de Gualtar, 4710-057 Braga, Portugal \\ b QOPNA, Department of Chemistry, University of Aveiro, 3810-193 Aveiro, Portugal
}

\section{A R T I C L E I N F O}

\section{Article history:}

Received 30 March 2014

Received in revised form 24 June 2014

Accepted 2 August 2014

Available online 17 August 2014

\section{Keywords:}

Dextrin

Injectable hydrogels

Structural analysis

MALDI-MS

SEC

\begin{abstract}
A B S T R A C T
The characterization of several commercial dextrins and the analysis of the potential of dextrin derived hydrogels for biomedical applications were performed in this work. The structural characterization of dextrins allowed the determination of the polymerization and branching degrees, which ranged from 6 to 17 glucose residues and 2 to 13\%, respectively. Tackidex, a medical grade dextrin was choosen for further characterization.

The combination of hydrogel with a dextrin nanogel and urinary bladder matrix was achieved without compromising the mechanical properties or microstructure. The encapsulation of cells, preserving its viability, confirms the biocompatibility of the injectable hydrogels, which have therefore great potential for biomedical applications.
\end{abstract}

(C) 2014 Published by Elsevier Ltd

\section{Introduction}

Dextrins are a class of low molecular weight carbohydrates produced by acid or/and enzymatic partial hydrolysis of starch or glycogen, thus exhibiting the $\alpha-(1 \rightarrow 4)$-Glc structure of amylose and the $\alpha-(1 \rightarrow 4)$ - and $\alpha-(1 \rightarrow 4,6)$-Glc branched structure of amylopectin, but with lower polymerization. The extent of hydrolysis is expressed in terms of "dextrose equivalent" (DE), a measure of the total reducing power (Chronakis, 1998; White, Hudson, \& Adamson, 2003). Dextrins with the same DE can display differences in terms of hygroscopicity, fermentability, viscosity, sweetness, stability, gelation, solubility, and bioavailability, which may be assigned to distinct structural features (Chronakis, 1998). Depending on the source of the native starch, as well as on the hydrolysis conditions, several types of dextrins can be obtained, displaying different properties that can be suited for specific applications.

Dextrins are an affordable raw material, generally regarded as safe (GRAS) (Alvani, Qi, \& Tester, 2011). It is a widely used material with a great variety of applications such as adhesives, foods,

\footnotetext{
* Corresponding author. Tel.: +351 253604 418; fax: +351 253678986 .

E-mail address: fmgama@deb.uminho.pt (F.M. Gama).

1 Contributed equally to this study.
}

textiles and cosmetics (Gonçalves, Moreira, Carvalho, Silva, \& Gama, 2014). Regarding biomedical applications, dextrin is yet relatively unexplored, being clinically used as a peritoneal dialysis solution that can also perform as a drug delivery solution (Peers \& Gokal 1998; Takatori et al., 2011) and as wound dressing agent (DeBusk \& Alleman, 2006). Although its limited number of current biomedical applications, dextrin exhibit a set of advantages that potentiates its use specifically in the biomaterials field. It is a biocompatible and non-immunogenic material, degradable in vivo by $\alpha$-amylases and its molecular weight ensures renal elimination avoiding tissue accumulation due to repeated administration (Hreczuk-Hirst, Chicco, German, \& Duncan, 2001; Moreira et al., 2010).

Hydrogels are three-dimensional, hydrophilic and polymeric networks that are highly hydrated and are receiving attention as scaffold materials (Drury \& Mooney, 2003; Hoffman, 2002). The relevance of injectable hydrogels for biomedical purposes has increased in the last years since they generally are biocompatible, biodegradable, exhibit mechanical and structural properties that resembles extracellular matrix, are processed under mild conditions and enable less invasive clinical procedures (Drury \& Mooney, 2003; Hoffman, 2002; Van Vlierberghe, Dubruel, \& Schacht, 2011). Hydrogels can be used alone or in combination with other components (e.g. cells, drugs, DNA, signalling molecules) (Jagur-Grodzinski, 2010; Peppas, Hilt, Khademhosseini, \& Langer, 2006). 
Several dextrin-based hydrogels, obtained by radical polymerization have been reported (Carvalho, Goncalves, Gil, \& Gama, 2007; Carvalho, Coimbra, \& Gama, 2009; Das, Das, Mandal, Ghosh, \& Pal, 2014; Moreira et al., 2010). Also, an oxidized dextrin hydrogel cross-linked with adipic acid dihydrazide was described by our group (Molinos, Carvalho, Silva, \& Gama, 2012). Adipic acid dihydrazide may be used to promote the reticulation of polysacharide-based hydrogels, such as oxidized dextran (Maia, Ferreira, Carvalho, Ramos, \& Gil, 2005), hyaluronic acid (Schramm et al., 2012);(Su, Chen, \& Lin, 2010), poly(aldehyde guluronate) (Bouhadir, Hausman, \& Mooney, 1999), among others. Despite the overall good biocompatibility, Schramm et al. (2012) reported a mild cytotoxic effect of the dihydrazide-reticulated hydrogels. However, information about the biocompatibility of non-crosslinked monomer is very scarce. This work provides a comprehensive structural characterization of several commercial dextrins, which were used to produce oxidized dextrin hydrogels reticulated with adipic acid dihydrazide. The cytotoxicity of the crosslinking agent was evaluated and compared with that of glutaraldehyde. The later is a widely used crosslinker, often considered cytotoxic (Huang-Lee, Cheung, \& Nimni, 1990; Mcpherson, Sawamura, \& Armstrong, 1986), but still used for reticulation of biomedical products (Furst \& Banerjee, 2005).

\section{Materials and methods}

\subsection{Materials}

All reagents used were of laboratory grade and purchased from Sigma-Aldrich, unless stated otherwise. Koldex 60 dextrin was a gift from Tate $\&$ Lyle. Tackidex was a gift from Roquette. Dextrins w28, w35, w60 and w80 were a gift from Avebe. Icodextrin and D4894 were obtained from Baxter and Sigma, respectively. All other chemicals and solvents used in this work were of the highest purity commercially available.

\subsection{Structural analysis of dextrins}

Neutral sugars were determined as alditol acetates as described by Nunes et al. (2012). The hydrolysis was performed with $\mathrm{H}_{2} \mathrm{SO}_{4}$ $1 \mathrm{M}$ at $100^{\circ} \mathrm{C}$ during $2.5 \mathrm{~h}$. Monosaccharides were reduced and acetylated and alditol acetate derivatives were analyzed by gas chromatography (GC) with a flame ionisation detector (Perkin Elmer, Clarus 400). The GC was equipped with a $30 \mathrm{~m}$ column DB225 (J\&W Scientific, Folsom, CA, USA) with i.d. and film thickness of $0.25 \mathrm{~mm}$ and $0.15 \mu \mathrm{m}$, respectively. The oven temperature program used was: initial temperature $200^{\circ} \mathrm{C}$, a rise in temperature at a rate of $40^{\circ} \mathrm{C} / \mathrm{min}$ until $220^{\circ} \mathrm{C}$, standing for $7 \mathrm{~min}$, followed by a rate of $20^{\circ} \mathrm{C} / \mathrm{min}$ until $230^{\circ} \mathrm{C}$ and maintain this temperature $1 \mathrm{~min}$. The injector and detector temperatures were, respectively, 220 and $230^{\circ} \mathrm{C}$. The flow rate of the carrier gas $\left(\mathrm{H}_{2}\right)$ was set at $1.7 \mathrm{~mL} / \mathrm{min}$.

\subsubsection{Methylation analysis}

Linkage analysis was carried out by methylation as described by Ciucanu and Kerek (1984). Dextrin samples (1-2 mg) were dissolved in $1 \mathrm{~mL}$ of anhydrous dimethylsulfoxide (DMSO). The sodium hydroxide pellets were ground with a pestle in a dry mortar to get a fine powder in a $\mathrm{N}_{2}$ atmosphere, then powdered $\mathrm{NaOH}$ (about $40 \mathrm{mg}$ ) was added and samples were methylated with $\mathrm{CH}_{3} \mathrm{I}(80 \mu \mathrm{L})$ during $20 \mathrm{~min}$. The methylated fractions were also carboxyl-reduced by a modification of the method described by Nunes et al. (2012). The methylated oligosaccharides were hydrolyzed with $2 \mathrm{M}$ TFA at $121^{\circ} \mathrm{C}$ for $1 \mathrm{~h}$, and then reduced and acetylated as previously described for neutral sugar analysis. The partially methylated alditol acetates (PMAA) were separated and analyzed by gas chromatography with mass spectrometry detector
(GC-MS) (Agilent Technologies 6890N). The GC was equipped with a DB-1 (J\&W Scientific, Folsom, CA, USA) capillary column (30 m length, $0.25 \mathrm{~mm}$ of internal diameter and $0.15 \mu \mathrm{m}$ of film thickness). The samples were injected in splitless mode (time of splitless $5 \mathrm{~min}$ ), with the injector operating at $220^{\circ} \mathrm{C}$, and using the following temperature program: $45^{\circ} \mathrm{C}$ for $5 \mathrm{~min}$ with a linear increase of $10^{\circ} \mathrm{C} / \mathrm{min}$ up to $140^{\circ} \mathrm{C}$, and standing for $5 \mathrm{~min}$ at this temperature, followed by linear increase of $0.5^{\circ} \mathrm{C} / \mathrm{min}$ up to $170^{\circ} \mathrm{C}$, and standing for $1 \mathrm{~min}$ at this temperature, followed by linear increase of $15^{\circ} \mathrm{C} / \mathrm{min}$ up to $280^{\circ} \mathrm{C}$, with further $5 \mathrm{~min}$ at $280^{\circ} \mathrm{C}$. The carrier gas was helium with a flow rate of $1.7 \mathrm{~mL} / \mathrm{min}$ and a column head pressure of 2.8 psi. The GC was connected to an Agilent 5973 mass quadrupole selective detector operating with an electron impact mode at $70 \mathrm{eV}$ and scanning the range $m / z 40-500$ in a $1 \mathrm{~s}$ cycle in a full scan mode acquisition.

\subsubsection{Matrix-assisted laser desorption/ionization mass spectrometry (MALDI-MS)}

The MALDI-MS mass spectra were acquired using MALDITOF/TOF Applied Biosystems 4800 Proteomics Analyzer (Applied Biosystems, Framingham, MA, USA) instrument equipped with a nitrogen laser emitting at $337 \mathrm{~nm}$. A volume of $4 \mu \mathrm{L}$ matrix (dithranol, $10 \mathrm{mg} / \mathrm{mL}$ in methanol and $0.1 \%$ TFA solution, or 2,5dihydroxybenzoic acid) were mixed with $2 \mu \mathrm{L}$ of phatlocyanine solution $(\sim 10 \mathrm{mg} / \mathrm{mL})$ and $1 \mu \mathrm{L}$ of this mixture was deposited on the MALDI plate. Spectra were subsequently acquired in the positive ion reflector mode using delayed extraction in the mass range between 600 and $4500 \mathrm{Da}$ with $\mathrm{ca}$. 1500 laser shots. Data acquisition was carried out using a 4000 Series Explorer data system (Applied Biosystems, Framingham, MA, USA) (Moreira, Coimbra, Nunes, Simões, \& Domingues, 2011).

\subsubsection{Size exclusion chromatography}

The determination of the average molecular weight $(M w)$ was performed by size exclusion chromatography (SEC) as described by Passos, Moreira, Domingues, Evtuguin, and Coimbra (2014). The samples were dissolved in an aqueous $0.1 \mathrm{M} \mathrm{NaNO}_{3}$ solution. A PL-GPC 110 chromatograph was equipped with a pre-column PLaquagel-OH $15 \mu \mathrm{m}$ and two SEC columns in series (PLaquagel-OH40 $15 \mu \mathrm{m}, 300 \times 7.0 \mathrm{~mm}$ and PLaquagel-OH60 $15 \mu \mathrm{m}, 300 \times 7.0 \mathrm{~mm}$ ). The pre-column, the SEC columns, the injection system, and the refractive index detector were maintained at $36^{\circ} \mathrm{C}$. The eluent (aqueous $0.1 \mathrm{M} \mathrm{NaNO}_{3}$ solution) was pumped at a flow rate of $0.9 \mathrm{~mL} / \mathrm{min}$. The analytical columns were calibrated with pullulan standards (Polymer Laboratories, UK) in the range of 5.8-1600 kDa.

\subsection{Cell culture}

Mouse embryo fibroblasts 3T3 (ATCC CCL-164) were grown in Dulbecco's modified Eagle's media supplemented with $10 \%$ newborn calf serum (Invitrogen, U.K.) and $1 \mu \mathrm{g} / \mathrm{mL}$ penicillin/streptavidin (CDMEM) at $37{ }^{\circ} \mathrm{C}$ in a $95 \%$ humidified air containing $5 \% \mathrm{CO}_{2}$. At $80 \%$ confluency, $3 \mathrm{~T} 3$ fibroblasts were harvested with $0.05 \%(\mathrm{w} / \mathrm{v})$ trypsin-EDTA and subcultivated in the same medium.

\subsubsection{Evaluation of adipic acid dihydrazide and glutaraldehyde induced-cytotoxicity}

The cytotoxicity of the un-cross linked adipic acid dihydrazide and glutaraldehyde was assessed in a 3T3 fibroblasts culture previously incubated for $24 \mathrm{~h}$ in a 24 -well plate $\left(2 \times 10^{4}\right.$ cell/well). After $0,24,48$ and $72 \mathrm{~h}$ of incubation, cell viability was determined by sulforhodamine B assay (Skehan et al., 1990). At different time points, cells were fixed in a methanol:acetic acid (1\%) solution at $-20^{\circ} \mathrm{C}$, during, at least, $30 \mathrm{~min}$. Then, a sulforhodamine B solution 
$(0.5 \% \mathrm{w} / \mathrm{v})$ in $1 \%$ acetic acid was added to the cells for $1.5 \mathrm{~h}$ at $37^{\circ} \mathrm{C}$. After several washes with $1 \%$ acetic acid solution, to remove the excess dye that did not bind to the proteins, the bound sulforhodamine $B$ was dissolved with a $10 \mathrm{mM}$ Tris solution. The absorbance at $540 \mathrm{~nm}$ was read in a multiplate reader (Bio-Tek, Synergy HT).

\subsubsection{Cell encapsulation in the hydrogel}

To perform cell encapsulation in the tackidex hydrogel, fibroblasts were mixed in the oxidised dextrin solution at a final density of $3.75 \times 10^{5}$ cell $/ \mathrm{mL}$ of hydrogel solution. Then, the adipic acid dihydrazide was added and the hydrogel was left to gelify at the bottom of the wells for $20 \mathrm{~min}$, after which the culture medium was added. The cell viability was assessed by Live and Dead assay (Invitrogen, UK) as indicated by the manufacturer with a slight modification. After $4.5 \mathrm{~h}$ of incubation the medium was replaced by DMEM without serum or phenol red and $200 \mu \mathrm{L}$ of a calcein AM $(2 \mu \mathrm{M})$ /ethidium homodimer-1 ( $4 \mu \mathrm{M})$ solution was added to each well and incubated for $1.5 \mathrm{~h}$. The hydrogels were visualized by fluorescence microscopy. The polystyrene plate and DMSO (20\%) were used as controls.

\subsection{Extraction of extracellular matrix from porcine bladder}

This procedure has been reported before (Freytes, Martin, Velankar, Lee, \& Badylak, 2008). Briefly, after harvesting, the bladders were washed to remove urine residues and stored in a $0.9 \%$ sodium chloride solution at $4{ }^{\circ} \mathrm{C}$ until removing the tunica serosa, muscularis externa, submucosa and muscularis mucosa. The urothelial cells were then removed in a solution of $1.0 \mathrm{M} \mathrm{NaCl}$, under agitation, in order to obtain the urinary bladder matrix consisting of the basement membrane of urothelial cells and subjacent lamina propria. The urinary bladder matrix was immersed in an aqueous solution of $0.1 \%(\mathrm{v} / \mathrm{v})$ of peracetic acid and $4 \%(\mathrm{v} / \mathrm{v})$ of ethanol, for $2 \mathrm{~h}$, under agitation, to promove the disinfection of the biomaterial. Peracetic acid residues were removed in two washes of 15 min in phosphate buffered saline (PBS) pH 7.4, followed by two washes of $15 \mathrm{~min}$ with sterile water. The urinary bladder matrix was freeze-dryed (LaboGene, Lynge, Denmark) until use.

\subsubsection{Enzymatic hydrolysis of urinary bladder matrix}

One gram of the lyophilized urinary bladder matrix was hydrolysed with $100 \mathrm{mg}$ of pepsin in $100 \mathrm{~mL}$ of $0.01 \mathrm{M}$ chloridric acid solution, during $48 \mathrm{~h}$, at $37^{\circ} \mathrm{C}$, under stirring. Pepsin inactivation was achieved by raising the $\mathrm{pH}$ to 8.0 .

\subsection{Preparation of hydrogels}

\subsubsection{Dextrin oxidation}

Dextrin oxidation was performed as described by Molinos et al. (2012). Briefly, aqueous solutions of dextrin ( $2 \% \mathrm{w} / \mathrm{v})$ were oxidized with a $2 \mathrm{~mL}$ sodium $m$-periodate solution, to yield the theoretical degree of oxidation of $40 \%$, at room temperature, with stirring, and in the dark. After $20 \mathrm{~h}$, the oxidation reaction was stoped by adding dropwise an equimolar amount of diethyleneglycol, to reduce any unreacted periodate. The resulting solution was dialyzed for 3 days against water, using a dialysis membrane with a molecular weight cut-off $1000 \mathrm{Da}$, and then lyophilized for 10 days.

\subsubsection{Determination of aldehyde groups by ${ }^{1} \mathrm{H}$ NMR analysis}

The degree of oxidation of oxidized dextrin is defined as the number of oxidized residues per 100 glucose residues, and was quantified using the tert-butylcarbazate (tBC) method, as described elsewhere (Bouhadir et al., 1999; Molinos et al., 2012). The ${ }^{1}$ H NMR spectra was used to determine the degree of oxidation, calculated as a peak area ratio in the NMR spectra according to the equation:
DO $(\%)=(X / Y) \times 100$ where, $X$ is the average integral corresponding to the peak at $\delta 7.3 \mathrm{ppm}$ and $Y$ is the average integral of the anomeric protons at $\delta 4.8 \mathrm{ppm}$ and $\delta 5.4 \mathrm{ppm}$.

\subsubsection{Preparation of dextrin-adipic acid dihydrazide hydrogels}

Oxidized dextrin was dissolved in PBS buffer (phosphate buffered saline) (30\% w/v) at room temperature and an adipic acid dihydrazide solution (prepared separately) was added at $5 \%$, in molar base, taking into account the number of glucose residues in the original dextrin. The crosslinking reaction was allowed to proceed during $2 \mathrm{~h}$.

\subsubsection{Preparation of hydrogel-nanogel combinations}

Dextrin nanogel was prepared as described by Molinos et al. (2012). The nanogel formation was confirmed by dynamic light scattering, as described previously (Carvalho et al., 2010; Goncalves \& Gama, 2008; Goncalves, Martins, \& Gama, 2007). Oxidized dextrin (oDex), DO 40\%, (30\% w/v) was dissolved in PBS (pH 7.4) or in a suspension of nanogel for approximately $16 \mathrm{~h}$ at room temperature. Then, the oxidized dextrin suspensions were mixed with $5 \%$ adipic acid dihydrazide (in molar basis taking into account the number of glucose residues in the original dextrin). The crosslinking was allowed to proceed at room temperature for about $2 \mathrm{~h}$.

\subsubsection{Preparation of hydrogel-urinary bladder matrix combinations}

Oxidized dextrin was dissolved in PBS buffer $\mathrm{pH} 7.4(30 \% \mathrm{w} / \mathrm{v})$ at room temperature. Urinary bladder matrix was dissolved in an adipic acid dihydrazide solution which was added at 5\%, in molar basis, taking into account the number of glucose residues in the original dextrin. The crosslinking reaction was allowed to proceed during $2 \mathrm{~h}$.

\subsection{Mechanical analysis}

Stress-strain experiments of crosslinked hydrogels and their combinations with a dextrin nanogel and urinary bladder matrix were assessed using a Shimadzu-AG-IS universal testing machine. Each hydrogel disc, with a superficial area ranging from 103 to $123 \mathrm{~mm}^{2}$ and a thickness $\approx 0.45 \mathrm{~mm}$, was placed between two parallel metallic circumferential plates, in order to promote a uniform distribution of the compressive force along the sample. The compressive experiments were carried out at a deformation rate of $0.5 \mathrm{~mm} / \mathrm{min}$ and at room temperature. The elastic modulus was determined by the average slope of the stress-strain curve over the strain range $0-20 \%$. For each condition samples in triplicate were analysed.

\subsection{Cryo-scanning electron microscopy (Cryo-SEM) analysis}

The topography and porosity of the hydrogels were studied by Cryo-SEM. Hydrogel samples were rapidly immersed in sub-cooled nitrogen (slush nitrogen) and transferred under vacuum to the cold stage of the preparation chamber. The frozen samples were fracturated, sublimated for $120 \mathrm{~s}$ at $-90^{\circ} \mathrm{C}$ (to partially sublimate water from the fractured hydrogel surface) and coated with $\mathrm{Au} / \mathrm{Pd}$ by sputtering for $50 \mathrm{~s}$ with a $12 \mathrm{~mA}$ current. Samples were then transferred into the SEM chamber and analysed at $-150^{\circ} \mathrm{C}$ using a High resolution Scanning Electron Microscope with X-ray Microanalysis and CryoSEM experimental facilities-JEOL JSM 6301F/Oxford INCA Energy 350/Gatan Alto 2500.

\subsection{Degradation of oxidized dextrin hydrogels}

After being prepared and weighted $\left(W_{i}\right)$, the hydrogels were immersed in PBS pH 7.4 (diffusion medium), and incubated at $37^{\circ} \mathrm{C}$. 
Table 1

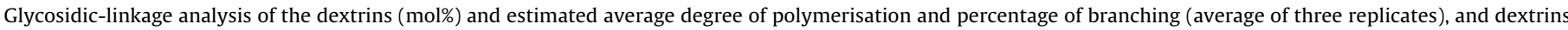
oxidation degree, gelation and degradation times of the hydrogels based on the different commercial dextrins.

\begin{tabular}{|c|c|c|c|c|c|c|c|c|}
\hline & w28 & w60 & D4894 & w35 & w80 & Tackidex & Icodextrin & Koldex $60^{a}$ \\
\hline T-Glcp & $25.3 \pm 1.2$ & $9.9 \pm 0.3$ & $17.2 \pm 2.4$ & $12.9 \pm 0.5$ & $14.8 \pm 0.3$ & $14.4 \pm 1.2$ & $19.1 \pm 2.1$ & 11.2 \\
\hline$(1 \rightarrow 4)-G l c p$ & $64.9 \pm 1.7$ & $86.2 \pm 0.6$ & $79.1 \pm 1.1$ & $84.7 \pm 0.7$ & $80.3 \pm 0.9$ & $73.7 \pm 1.9$ & $68.7 \pm 3.9$ & 85.3 \\
\hline$(1 \rightarrow 6)-$ Glcp & - & - & - & - & - & $3.3 \pm 0.5$ & $0.3 \pm 0.1$ & - \\
\hline$(1 \rightarrow 4,6)-\mathrm{Glcp}$ & $9.8 \pm 2.9$ & $3.9 \pm 0.3$ & $3.7 \pm 0.5$ & $2.4 \pm 0.2$ & $4.9 \pm 1.2$ & $8.6 \pm 0.8$ & $11.9 \pm 1.6$ & 3.5 \\
\hline $\mathrm{DP}$ & 6 & 17 & 7 & 10 & 10 & 16 & 13 & 13 \\
\hline Branching (\%) & 11 & 4 & 4 & 2 & 5 & 9 & 13 & 4 \\
\hline Oxidation degree (\%) & $28.9 \pm 1.59$ & $28.7 \pm 2.80$ & - & $27.6 \pm 1.09$ & $30.1 \pm 3.58$ & $31.7 \pm 1.95$ & $30.9 \pm 1.56$ & $33.2 \pm 2.82$ \\
\hline Gelation time (min) & $2-5$ & $<1$ & - & 5 & $<1$ & $1-5$ & $1-2$ & $<1$ \\
\hline Degradation time (days) & 1 & 1 & - & 1 & 1 & 1 & 1 & 25 \\
\hline
\end{tabular}

a Data from Carvalho et al. (2009).

At regular intervals, they were removed from the solutions, blotted with filter paper, weighed $\left(W_{t}\right)$ and returned to the same container. The buffer solution was replaced at each measurement. The percentage of mass loss was determined using the equation: mass loss $(\%)=100-\left[\left(W_{t} / W_{i}\right) \times 100\right]$.

\subsection{Hydrogel behaviour in human blood and plasma}

Blood samples from three different donners were collected. From each sample, plasma was collected by centifugation at $500 \times g$ for $5 \mathrm{~min}$. After being prepared, the hydrogel were injected in the blood and plasma samples.

\subsection{Data analysis}

Data are presented as mean \pm SD of the indicated number $(n)$ of assays. Statistical analysis was performed using the variance analysis method (ANOVA) by GraphPad PRISM 4.0c (GraphPad software, USA). Differences were considered significant when $p<0.05$.

\section{Results and discussion}

\subsection{Structural dextrin analysis}

The dextrins used in this work are commercially available products. These materials are not sufficiently characterized, and as it will be shown, some of the available information is equivocal. In this work, we aimed at correlating the dextrin's structural differences with the properties of the derived hydrogels. All dextrin's analysed were composed only by glucose, as confirmed by sugar methylation analysis, which revealed the presence of $(1 \rightarrow 4)-$, terminally-, and $(1 \rightarrow 4,6)$-linked Glcp residues in all samples (Table 1$)$.

The amount of terminal residues in the main backbone can be estimated as the difference between the total terminal residues and those corresponding to the branching points. Taking this into account, the average degree of polymerisation of the dextrins can be estimated as the abundance of all residues divided by the abundance of terminal residues in the main backbone. This allows the calculation of an average degree of polymerisation ranging from 6 to 17 glucose residues (Table 1 ). The methylation analysis also showed that the samples had a degree of branching between 2 and $13 \%$. These values were obtained by estimating the percentage of branching residues in the main backbone, assuming that each branching point contains at least one additional glucose residue attached. The dextrin with the lowest degree of polymerisation (w28) had also one of the highest percentages of branching points, thus this is a small and highly branched dextrin. Conversely, the dextrin w60 showed the highest average degree of polymerisation (17 residues) with a degree of branching of only $4 \%$. The abundance of terminal residues exceeded three times that of branched ones in the case of $w 28, w 60$, and $w 80$, and five times in the case of D4894 and w35. Probably, the 3:1 proportion results from the presence of linear structures (bearing 1 terminal and no branching residues) mixed in similar amounts with others with one branching point, while the 5:1 ratio for the other samples should translate the presence of five times more linear structures than branched ones.

The SEC analysis showed that the apparent $M w$ ranged from $3.4 \mathrm{kDa}$ for D4894 to $16.6 \mathrm{kDa}$ for Icodextrin (Table 2). These values were assessed using pullulan standards. This linear molecule was chosen as a standard due to it's structural similarity to dextrin, since both are glucose polymers linked by $\alpha-(1 \rightarrow 4)$ bonds. Moreover, dextrins have a low branching degree. Indeed, as discussed above, a large fraction of the dextrin molecules are linear and thus comparable to pullulan. The polydispersity $(\mathrm{Mw} / \mathrm{Mn})$ was very high in all cases, varying from 1.8 for D4894 and Icodextrin to 5.9 for Koldex 60 , suggesting that the samples are very heterogeneous. Further, the $M w$ estimated by SEC correspond to DP values much higher than those estimated by the glycosidic-linkage analysis (Table 1 ). The higher molecular branching provides higher hydrodynamic volumes and, consequently, higher apparent molecular weight. This is in accordance with the higher Mw estimated by SEC for Icodextrin and w28, with $13 \%$ and $11 \%$ branching, respectively. On the contrary, sample D4894, with a DP estimated by linkage-analysis comparable with w28 but with a lower \% of branching (4\%), showed a three times lower estimated $M w$. Also, although bearing an apparent $M w$ similar to w28 (9 kDa), Koldex 60 had a twofold higher DP and a threefold lower \% of branching, showing the dependence of the SEC estimation of the $M w$ on the molecular branching.

The presence of low molecular weight molecules in the dextrin samples was confirmed by MALDI-MS analysis (Table 3). Oligosacharides ranging from DP 5 to DP 12 were detected using this technique. In the case of D4894 and w35, it was also possible to identify dextrins with up to DP18, confirming their higher polydispersity. Noteworthy, MALDI-MS analysis of Koldex 60 after ultrafiltration through a $3 \mathrm{kDa} M w \mathrm{CO}$ membrane revealed that most of the material crossed the membrane. This was unexpected, considering the molecular weight estimated for this dextrin by SEC analysis ( $9 \mathrm{kDa}$ ). Furthermore, the residue collected from the retentate in the ultrafiltration revealed the same DP composition as the filtrate, as checked by MALDI-MS analysis. These results show very clearly that the Koldex 60 dextrins are indeed composed of low

Table 2

Estimation of the average degree of polymerization (DP) by SEC.

\begin{tabular}{lrllr}
\hline Dextrins & \multicolumn{1}{c}{$M w$} & $M n$ & $M w / M n$ & DP \\
\hline w28 & 9040 & 2900 & 3.1 & 55 \\
w60 & 10,600 & 3000 & 3.5 & 65 \\
D4894 & 3400 & 1900 & 1.8 & 21 \\
w35 & 5940 & 2490 & 2.4 & 37 \\
w80 & 8320 & 2900 & 2.9 & 51 \\
Tackidex & 4500 & 2040 & 2.2 & 28 \\
Icodextrin & 16,560 & 8930 & 1.8 & 102 \\
Koldex 60 & 9200 & 1550 & 5.9 & 57
\end{tabular}


Table 3

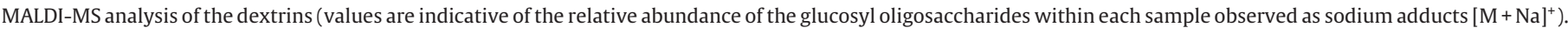

\begin{tabular}{|c|c|c|c|c|c|c|c|c|c|c|c|c|c|c|c|c|}
\hline$m / z[\mathrm{M}+\mathrm{Na}]^{+}$ & $\begin{array}{l}\text { DP3 } \\
527\end{array}$ & $\begin{array}{l}\text { DP4 } \\
689\end{array}$ & $\begin{array}{l}\text { DP5 } \\
851\end{array}$ & $\begin{array}{l}\text { DP6 } \\
1013\end{array}$ & $\begin{array}{l}\text { DP7 } \\
1175\end{array}$ & $\begin{array}{l}\text { DP8 } \\
1337\end{array}$ & $\begin{array}{l}\text { DP9 } \\
1499\end{array}$ & $\begin{array}{l}\text { DP10 } \\
1661\end{array}$ & $\begin{array}{l}\text { DP11 } \\
1823\end{array}$ & $\begin{array}{l}\text { DP12 } \\
1985\end{array}$ & $\begin{array}{l}\text { DP13 } \\
2147\end{array}$ & $\begin{array}{l}\text { DP14 } \\
2309\end{array}$ & $\begin{array}{l}\text { DP15 } \\
2471\end{array}$ & $\begin{array}{l}\text { DP16 } \\
2633\end{array}$ & $\begin{array}{l}\text { DP17 } \\
2795\end{array}$ & $\begin{array}{l}\text { DP18 } \\
2957\end{array}$ \\
\hline w28 & 100 & 95 & 73 & 55 & 35 & 25 & 18 & 13 & 10 & 4 & 3 & 2 & & & & \\
\hline w60 & 55 & 100 & 93 & 78 & 62 & 45 & 48 & 28 & 20 & 20 & 12 & 8 & 5 & 4 & 3 & \\
\hline D4894 & 74 & 96 & 100 & 85 & 76 & 71 & 53 & 50 & 41 & 28 & 29 & 15 & 13 & 6 & 6 & 6 \\
\hline w35 & 79 & 99 & 100 & 74 & 64 & 45 & 35 & 25 & 20 & 16 & 14 & 8 & 8 & 3 & 2 & 2 \\
\hline w80 & 86 & 100 & 100 & 84 & 67 & 49 & 35 & 23 & 20 & 10 & 9 & 5 & 5 & & & \\
\hline Tackidex & 57 & 100 & 98 & 80 & 72 & 50 & 35 & 24 & 20 & 10 & 10 & 6 & 4 & 2 & & \\
\hline Icodextrin & 100 & 26 & 11 & 6 & 7 & 8 & 16 & 5 & 5 & 5 & 4 & 4 & & & & \\
\hline Koldex 60 & 100 & 68 & 30 & 25 & 20 & 15 & 10 & 8 & 4 & 2 & 1 & & & & & \\
\hline
\end{tabular}

molecular weight compounds, with DPs comparable to those estimated by methylation analysis. These results suggest that the SEC analysis does not in this case provide an accurate estimation of the molecular weight, probably due to aggregation of the poor water soluble dextrin molecules.

The different dextrins were used to prepare hydrogels. These were obtained using oxidized dextrins reticulated with adipic acid dihydrazide, as comprehensively described in a previous work (Molinos et al., 2012). Despite the structural differences concerning the polymerization and branching degrees of the dextrins, the hydrogels produced from the different dextrins performed fairly similarly in terms of gelation and degradation time, exception made for Koldex 60, which exhibited a very slow degradation rate combined with a faster gelation. Since no significant differences, apart from Koldex, were observed between the dextrin hydrogels, Tackidex, was choosen for further characterization. This is a medical grade dextrin, therefore better suited for the aimed development of biomedical applications. As compared to some of the other dextrins, it bears a suitable gelification rate, convenient for its use as an injectable device, easy to handle by the surgeon during the procedures.

\subsection{Evaluation of adipic acid dihydrazide and glutaraldehyde induced-cytotoxicity}

As can be seen in Fig. 1A, a marked cytotoxic effect of adipic acid dihydrazide related with cell death - at e.g.72 h - can be observed only for the highest concentrations tested $(2-4 \% \mathrm{w} / \mathrm{v})$. In the production of the hydrogel a concentration of about $1 \%$ $(\mathrm{w} / \mathrm{v})$ is used, which according to the results induce an inhibitory effect on cell proliferation. However, the actual free dihydrazide concentration in the hydrogel is much lower, since the dihydrazide quickly reacts with the aldehyde groups of dextrin chains. When comparing the two crosslinkers (Fig. 1B), it can be seen that glutaraldehyde has a much higher cytotoxicity. Some studies on the biocompatibility of glutaraldehyde-crosslinked polymers - chitosan-based scaffolds for cartilage tissue (Hoffmann, Seitz, Mencke, Kokott, \& Ziegler, 2009), chitosan/poly(vinyl alcohol) blends (Costa, Barbosa-Stancioli, Mansur, Vasconcelos, \& Mansur, 2009), collagen scaffolds (Lee, Grodzinsky, \& Spector, 2001; Sheu, Huang, Yeh, \& Ho, 2001) - have demonstrated a satisfactory in vitro response in terms of cell adhesion and proliferation. On the other hand, other works reported some cytotoxicity, as in the case of
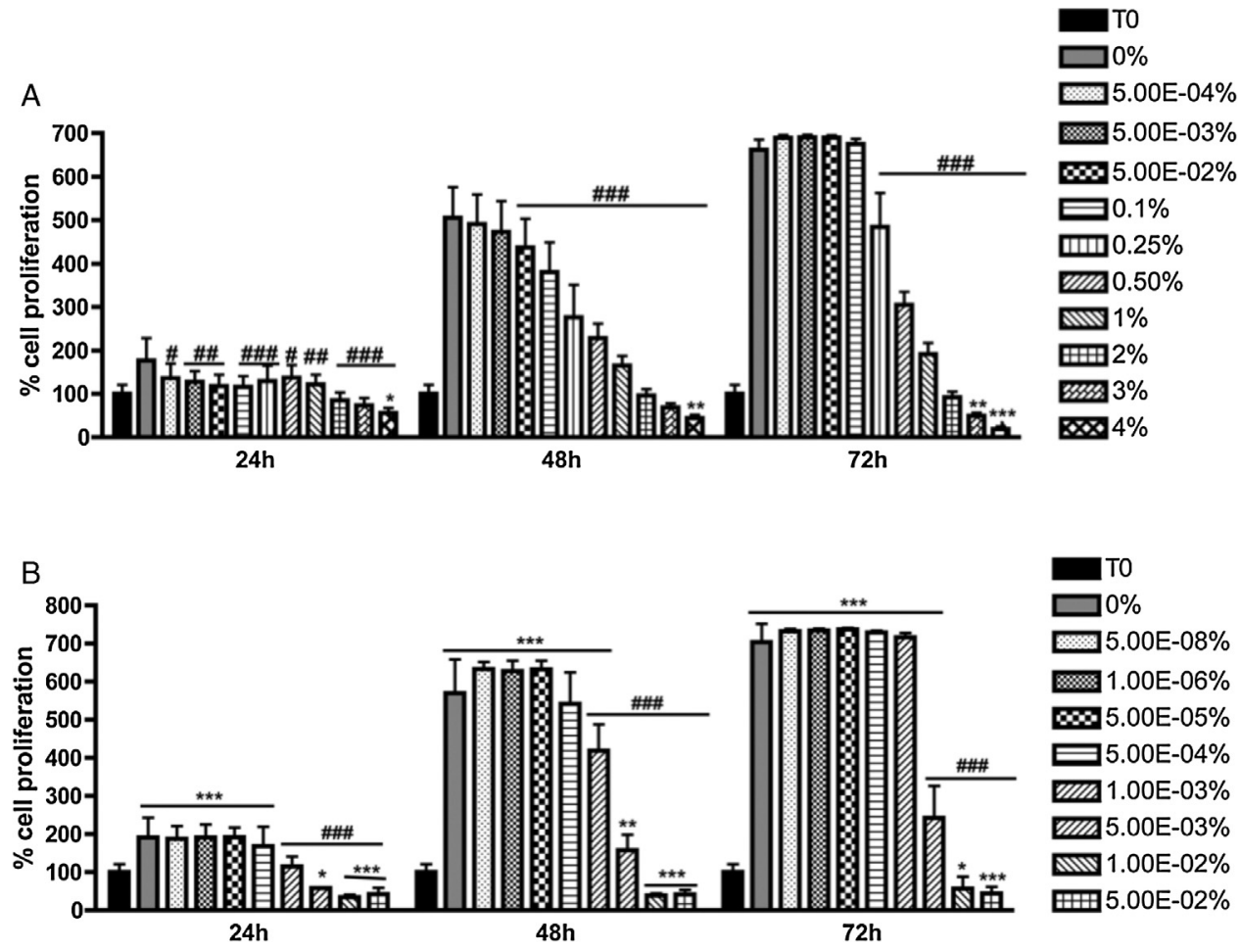

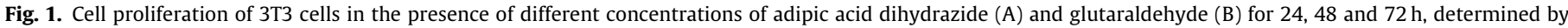

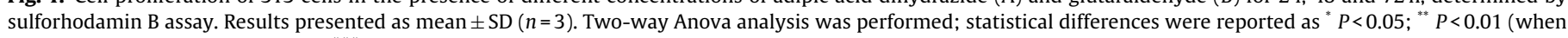
compared with the T0 control) and ${ }^{\# \#} P<0.001$ (when compared with the DMEM control-0\%). 

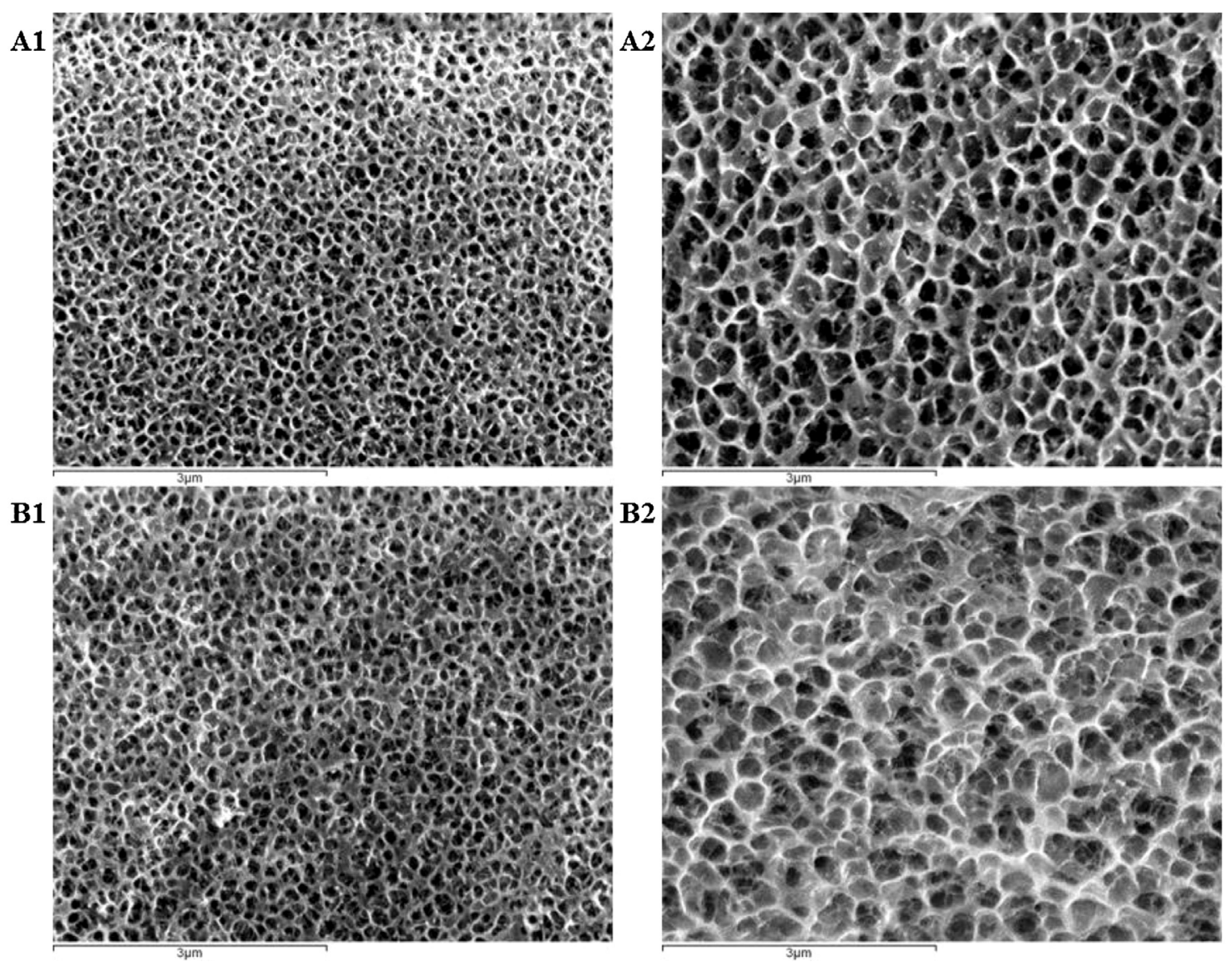

Fig. 2. Cryo-SEM images from cross-sections of hydrogel (A) and hydrogel-urinary bladder matrix (B) before (1) and after (2) immersion on distilled water for $6 \mathrm{~h}$.

glutaraldehyde-crosslinked collagen membranes (Marinucci et al., 2003), or in the study of Furst and Banerjee (2005), used as a commercial biomedical glue containing glutaraldehyde, which induces inflammation, edema and necrosis, in vivo.

Overall, our results showed that dihydrazide-induced cell death occurs only at a concentration 300 times higher the one of glutaraldehyde. Genipin is reputed a highly biocompatible crosslinker. As shown in a study comparing the cytotoxicity of genipin and glutaraldehyde, the former showed toxic effects at concentrations 10000 times superior to those of glutaraldehyde. Although genipin and adipic acid dihydrazide were not directly compared in this study, it can be concluded that adipic acid dihydrazide is more cytotoxic than genipin (by about 30 fold) but much less than glutaraldehyde (Sung, Huang, Huang, \& Tsai, 1999).

\subsection{Characterization of nanogel and urinary bladder matrix combined dextrin hydrogels}

The oxidized dextrin hydrogel was combined with a dextrin nanogel and with an enzymatic hydrolizate of porcine bladder derived extracellular matrix, to improve its functional and bioactive properties. The dextrin nanogel, previously developed in our group (Goncalves \& Gama, 2008; Goncalves et al., 2007) was obtained by grafting hydrophobic molecules to the dextrin backbone, which self-assembles in water, originating nanoparticles with a hydrophobic core. The incorporation of proteins (Carvalho et al., 2010; Molinos et al., 2012) and hydrophobic drugs (Gonçalves, Pereira, Schellenberg, Coutinho, \& Gama, 2012) was successfully achieved using these nanogels. The extracellular matrix is produced by cells of each tissue and organ and consists of 3D structure composed generally by type I collagen, glycosaminoglycans, fibronectin, laminin and a variety of growth factors. It is a natural scaffold for tissue regeneration, thus highly valuable for regenerative medicine applications (Badylak, Taylor, \& Uygun, 2011; Benders et al., 2013). The structural characterization, mechanical analysis and degradation profiles of the combined materials (hydrogel, urinary bladder matrix and nanogels) are described below.

\subsubsection{Cryo-SEM analysis}

The morphology of the oxidized dextrin hydrogels as well as their combination with urinary bladder matrix was assessed by Cryo-SEM analysis (Fig. 2). A characteristic three-dimentional structure is observed for all samples, with a network of interconnective pores, often found in this kind of materials (Annabi, Mithieux, Weiss, \& Dehghani, 2009; Zhang \& Chu, 2002; Zhang, Tang, Bowyer, Eisenthal, \& Hubble, 2005). The pore diameter is highly heterougeneous within samples and can vary between 200 and $400 \mathrm{~nm}$. Despite the pore size heterogeneity, when comparing the un-swollen hydrogels with the ones immersed in water for $6 \mathrm{~h}$, an increase in the pore size can be observed.

\subsubsection{Degradation profiles of combined dextrin hydrogels.}

Fig. 3 depicts the mass loss profiles of oxidized dextrin hydrogel incubated in PBS ( $\mathrm{pH} 7.4)$ at $37^{\circ} \mathrm{C}$, alone and combined with dextrin nanogel $(1 \mathrm{mg} / \mathrm{mL})$ and urinary bladder matrix $(4 \mathrm{mg} / \mathrm{mL})$. For all combinations, a similar degradation profile can be observed, with an initial swelling phase during the first $4 \mathrm{~h}$, followed by gradual mass loss until complete dissolution (12-24h). The swelling phase is caused by the hydrolysis of the hydrazone bonds 


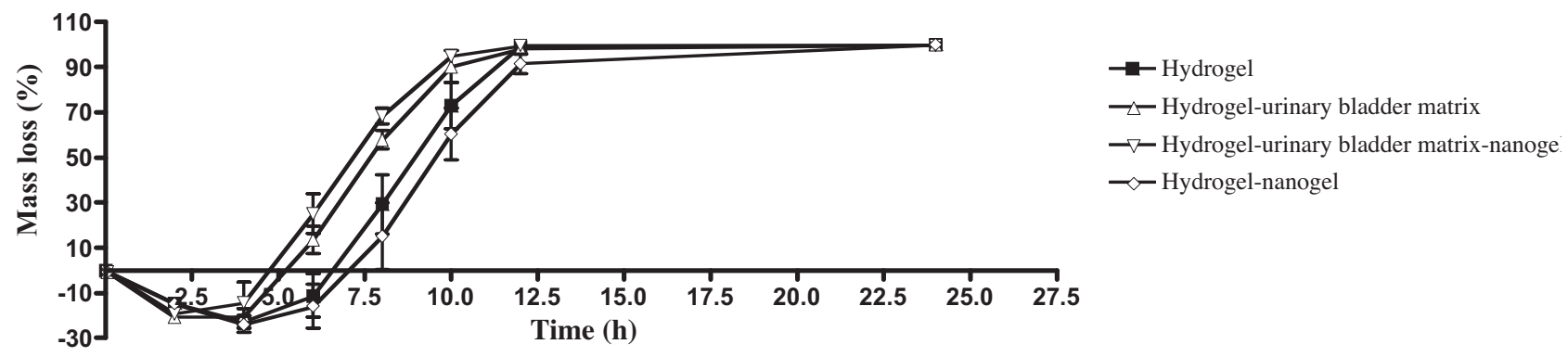

Fig. 3. Degradation profiles of hydrogel and combinations with nanogel and urinary bladder matrix in $\mathrm{PBS}(\mathrm{pH} 7.4)$ at $37^{\circ} \mathrm{C}$.

(Molinos et al., 2012) and osmotic effects. The combination with urinary bladder matrix seems to results in a slightly higher degradation rate. This is probably because the peptides may react with the aldehyde groups of oxidized dextrin, lowering the cross-linking sites. However, through Cryo-SEM analysis, no structural differences were detected between hydrogel-urinary bladder matrix combination and hydrogel alone. The degradation seems to occur by a bulk erosion process, that is, by permeation of water into the matrix, leading to the network desintegration by a non-linear degradation profile (von Burkersroda, Schedl, \& Gopferich, 2002).

Hydrogels made of different polysaccharides using a similar approach have been reported. Oxidized hyaluronic acid hydrogels reticulated with adipic acid dihydrazide take from 3 to 30 days to get fully solubilized, depending on the dihydrazide concentration used (Su et al., 2010). Maia et al. (2005) described an oxidized dextran hydrogel cross-linked with adipic acid dihydrazide, which degrades in 10 to 23 days, also depending on the crosslinker concentration. The relatively quick degradation rate of the Tackidex hydrogel is convenient for some applications, such as for instance bone regeneration. In this particular case, the hydrogel is aimed to perform as an injectable carrier of hydroxyapatite granules and as a drug delivery system. The incorporation of urinary bladder matrix brings bioactivity to this formulation, which proves to be very effective in supporting cell adhesion and proliferation. The results obtained using formulations of tackidex hydrogels with hydroxyapatite granules, in bone regeneration studies in the rabbit (data not shown), demonstratates that the in vivo degradation is actually slower than in vitro yet compatible with a swift cell invasion. In the case of hydrogels with much slower degradation rates, the regeneration is hampered by the physical presence of the reticulated matrix, which avoids the cell proliferation in the bone defect. The very promising results obtained in vivo using the tackidex formulations, namely demonstrating the suitability of the observed degradation rates, will be published elsewhere.

\subsubsection{Mechanical analysis}

The compressive mechanical properties of oxidized dextrin hydrogel as well as of its combinations with dextrin nanogel $(1 \mathrm{mg} / \mathrm{mL})$ and urinary bladder matrix $(4 \mathrm{mg} / \mathrm{mL})$ showed similar stress-strain profiles. Mechanical measurements revealed that samples have a linear behaviour at deformations below 30\% and for higher strains the stress-strain curve became non-linear, which indicates that plastic deformation occurred in the hydrogel samples.

The compression modulus calculated at 20\% deformation for hydrogel alone was $0.023 \pm 0.012 \mathrm{MPa}$, similar to the value found for hydrogel-nanogel, hydrogel-urinary bladder matrix and hydrogel-nanogel-urinary bladder matrix combinations. Finally, the maximum compressive strength also showed a similar behaviour between the different samples. These results suggest that the incorporation of the different materials (nanogel and urinary bladder matrix) in the hydrogel matrix does not significantly change its mechanical performance and corroborates the Cryo-SEM results (Fig. 2), which shows that different samples had similar microstructure. Since the compressive modulus of hydrogels is directly proportional to the intermolecular cross-link density (Anseth, Bowman, \& Brannon-Peppas, 1996), the incorporation of dextrin nanogel, urinary bladder matrix or both does not appear to substantially interfer in the linkages between aldehydes and the dihydrazide. The compressive properties of our biomaterial were comparable to those of others reported in literature (Liu \& ChanPark, 2009; Tan \& Hu, 2012).

\subsection{Interaction with cells and blood}

In order to mimick the clinical procedure, the percursor solutions were mixed and transferred to a syringe were the gelificatin started. Then, the hydrogel was injected in the human blood and plasma solutions. Hydrogel gelified as usual, no differences arising from the contact with the blood/plasma proteins.

The potential of oxidized dextrin hydrogel as a cell carrier system was tested by live and dead assay of encapsulated mouse embryo 3T3 fibroblasts. A cell suspension was mixed in the solution of oxidized tackidex dextrin and reticulated with the adipic acid dihydrazide. The hydrogel was left gelifying in a multiwell plate and after $20 \mathrm{~min}$ culture medium was added. The live and dead fluorescence was analysed after $6 \mathrm{~h}$ of encapsulation. The same analysis was performed for the hydrogel combined with urinary bladder matrix. The majority of the cells that were kept encapsulated in the tackidex hydrogel $(98.2 \pm 1.9 \%)$ and hydrogel with urinary bladder matrix $(99.5 \pm 0.7 \%)$ were calcein positive (viable) cells. The results confirm the cytocompatibility of oxidized dextrin hydrogel, since the cells could endure the cross-linking stage, thus becoming a suitable platform for the incorporation of cells to be used for tissue engineering purposes. Indeed, preliminary studies on the encapsulation of mesenchymal stem cells for in vivo administration demonstrate that the cells keep their viability; thus, this hydrogel may be a valuable platform for cell therapy applications.

\section{Conclusions}

The structural characterization showed that dextrins from different sources displayed structural differences, namely regarding chain length and branching degree. The molecular weight of dextrins is much lower than the values often reported in the literature, commonly obtained by SEC. However, these differences do not seem to influence the gelation and degradation of the hydrogels. The combination with a dextrin nanogel and urinary bladder matrix was successfully achieved. The cytotoxicity of the free adipic acid dihydrazide was evaluated and only a mild inhibitory effect on cell proliferation was observed for the concentration used in the hydrogel crosslinking. The biocompatibility of oxidized dextrin hydrogels was attested through the encapsulation of cells, which were able to endure the gelation process. Overall, these results demonstrate 
that the injectable dextrin hydrogels are a promising choice for biomedical applications, namely for regenerative medicine and cell therapies.

\section{Acknowledgments}

D.M.S. was supported by the grant SFRH/BD/64571/2009 from Fundação para a Ciência e Tecnologia (FCT), Portugal. We thank FCT funding through EuroNanoMed ENMED/0002/2010. The authors acknowledge the funding from QREN ("Quadro de Referência Estratégica Nacional”) and ADI ("Agência de Inovação") through the project Norte-07-0202-FEDER-038853.

QOPNA research unit is funded by Fundação para a Ciência e a Tecnologia (FCT, Portugal, European Union, QREN, FEDER, and COMPETE (project PEst-C/QUI/UI0062/2013; FCOMP01-0124-FEDER-037296), and the Portuguese National Mass Spectrometry Network, and Project PTDC/QUI-QUI/100044/2008. Ana S. P. Moreira and Cláudia Nunes thanks FCT the grants (SFRH/BD/80553/2011; SFRH/BPD/46584/2008). The authors thank Prof. Dmitry V. Evtuguin for the use of SEC equipment.

\section{References}

Alvani, K., Qi, X., \& Tester, R. F. (2011). Use of carbohydrates, including dextrins, for oral delivery. Starch-Starke, 63(7), 424-431.

Annabi, N., Mithieux, S. M., Weiss, A. S., \& Dehghani, F. (2009). The fabrication of elastin-based hydrogels using high pressure $\mathrm{CO}(2)$. Biomaterials, 30(1), 1-7.

Anseth, K. S., Bowman, C. N., \& Brannon-Peppas, L. (1996). Mechanical properties of hydrogels and their experimental determination. Biomaterials, 17(17), 1647-1657.

Badylak, S. F., Taylor, D., \& Uygun, K. (2011). Whole-organ tissue engineering: Decellularization and recellularization of three-dimensional matrix scaffolds. Annual Review of Biomedical Engineering, 13, 27-53.

Benders, K. E., van Weeren, P. R., Badylak, S. F., Saris, D. B., Dhert, W. J., \& Malda, J. (2013). Extracellular matrix scaffolds for cartilage and bone regeneration. Trends in Biotechnology, 31(3), 169-176.

Bouhadir, K. H., Hausman, D. S., \& Mooney, D. J. (1999). Synthesis of cross-linked poly(aldehyde guluronate) hydrogels. Polymer, 40(12), 3575-3584.

Carvalho, J., Goncalves, C., Gil, A. M., \& Gama, F. M. (2007). Production and characterization of a new dextrin based hydrogel. European Polymer Journal, 43(7), 3050-3059.

Carvalho, J. M., Coimbra, M. A., \& Gama, F. M. (2009). New dextrin-vinylacrylate hydrogel: Studies on protein diffusion and release. Carbohydrate Polymers, 75(2), 322-327.

Carvalho, V., Castanheira, P., Faria, T. Q., Gonçalves, C., Madureira, P., Faro, C., Domingues, L., Brito, R. M. M., Vilanova, M., \& Gama, M. (2010). Biological activity of heterologous murine interleukin-10 and preliminary studies on the use of a dextrin nanogel as a delivery system. International Journal of Pharmaceutics, $400(1-2), 234-242$

Chronakis, I. S. (1998). On the molecular characteristics, compositional proper ties, and structural-functional mechanisms of maltodextrins: A review. Critical Reviews in Food Science and Nutrition, 38(7), 599-637.

Ciucanu, I., \& Kerek, F. (1984). A simple and rapid method for the permethylation of carbohydrates. Carbohydrate Research, 131(2), 209-217.

Costa, E. S., Barbosa-Stancioli, E. F., Mansur, A. A. P., Vasconcelos, W. L., \& Mansur, H. S. (2009). Preparation and characterization of chitosan/poly(vinyl alcohol) chemically crosslinked blends for biomedical applications. Carbohydrate Polymers 76(3), 472-481

Das, D., Das, R., Ghosh, P., Dhara, S., Panda, A. B., \& Pal, S. (2013). Dextrin cross linked with poly(HEMA): A novel hydrogel for colon specific delivery of ornidazole. RSC Advances, 3(47), 25340-25350.

Das, D., Das, R., Mandal, J., Ghosh, A., \& Pal, S. (2014). Dextrin crosslinked with poly(lactic acid): A novel hydrogel for controlled drug release application. Jour nal of Applied Polymer Science, 131(7), n/a-n/a14.

DeBusk A., \& Alleman T. (2006). Method for preparing medical dressings. Vol US2006/0018955 A1. Powell, TN: DeRoyal Industries, Inc.

Drury, J. L., \& Mooney, D. J. (2003). Hydrogels for tissue engineering: Scaffold design variables and applications. Biomaterials, 24(24), 4337-4351.

Freytes, D. O., Martin, J., Velankar, S. S., Lee, A. S., \& Badylak, S. F. (2008). Preparation and rheological characterization of a gel form of the porcine urinary bladder matrix. Biomaterials, 29(11), 1630-1637.

Furst, W., \& Banerjee, A. (2005). Release of glutaraldehyde from an albuminglutaraldehyde tissue adhesive causes significant in vitro and in vivo toxicity. Annals of Thoracic Surgery, 79(5), 1522-1529.

Goncalves, C., \& Gama, F. M. (2008). Characterization of the self-assembly process of hydrophobically modified dextrin. European Polymer Journal, 44(11), 3529-3534.

Goncalves, C., Martins, J. A., \& Gama, F. M. (2007). Self-assembled nanoparticles of dextrin substituted with hexadecanethiol. Biomacromolecules, 8(2), 392-398.
Gonçalves, C., Moreira, S. M., Carvalho, V., Silva, D. M., \& Gama, F. M. (2014). Dextrin for biomedical applications. In M. Mishra (Ed.), Encyclopedia of biomedical polymers and polymeric biomaterials. New York, NY: Taylor and Francis (in press).

Gonçalves, C., Pereira, P., Schellenberg, P., Coutinho, P. J., \& Gama, F. M. (2012). Selfassembled dextrin nanogel as curcumin delivery system. Journal of Biomaterials and Nanobiotechnology, 3(2), 178-184.

Hoffman, A. S. (2002). Hydrogels for biomedical applications. Advanced Drug Delivery Reviews, 54(1), 3-12.

Hoffmann, B., Seitz, D., Mencke, A., Kokott, A., \& Ziegler, G. (2009). Glutaraldehyde and oxidised dextran as crosslinker reagents for chitosan-based scaffolds for cartilage tissue engineering. Journal of Materials Science-Materials in Medicine, 20(7), 1495-1503.

Hreczuk-Hirst, D., Chicco, D., German, L., \& Duncan, R. (2001). Dextrins as potentia carriers for drug targeting: Tailored rates of dextrin degradation by introduction of pendant groups. International Journal of Pharmaceutics, 230(1-2), 57-66.

Huang-Lee, L. L., Cheung, D. T., \& Nimni, M. E. (1990). Biochemical changes and cytotoxicity associated with the degradation of polymeric glutaraldehyde derived crosslinks. Journal of Biomedical Materials Research, 24(9), 1185-1201.

Jagur-Grodzinski, J. (2010). Polymeric gels and hydrogels for biomedical and pharmaceutical applications. Polymers for Advanced Technologies, 21(1), 27-47.

Lee, C. R., Grodzinsky, A. J., \& Spector, M. (2001). The effects of cross-linking of collagen-glycosaminoglycan scaffolds on compressive stiffness, chondrocytemediated contraction, proliferation and biosynthesis. Biomaterials, 22(23), 3145-3154.

Liu, Y., \& Chan-Park, M. B. (2009). Hydrogel based on interpenetrating polyme networks of dextran and gelatin for vascular tissue engineering. Biomaterials, 30(2), 196-207.

Maia, J., Ferreira, L., Carvalho, R., Ramos, M. A., \& Gil, M. H. (2005). Synthesis and characterization of new injectable and degradable dextran-based hydrogels. Polymer 46(23), 9604-9614.

Marinucci, L., Lilli, C., Guerra, M., Belcastro, S., Becchetti, E., Stabellini, G., Calvi, E. M. \& Locci, P. (2003). Biocompatibility of collagen membranes crosslinked with glutaraldehyde or diphenylphosphoryl azide: An in vitro study. Journal of Biomedical Materials Research A, 67A(2), 504-509.

Mcpherson, I. M., Sawamura, S., \& Armstrong, R. (1986). An examination of the biologic response to injectable, glutaraldehyde cross-linked collagen implants. Journal of Biomedical Materials Research, 20(1), 93-107.

Molinos, M., Carvalho, V., Silva, D. M., \& Gama, F. M. (2012). Development of a hybrid dextrin hydrogel encapsulating dextrin nanogel as protein delivery system. Biomacromolecules, 13(2), 517-527.

Moreira, A. S. P., Coimbra, M. A., Nunes, F. M., Simões, J., \& Domingues, M. R. r. M. (2011). Evaluation of the effect of roasting on the structure of coffee galactomannans using model oligosaccharides. Journal of Agricultural and Food Chemistry, 59(18), 10078-10087

Moreira, S., da Costa, R. M. G., Guardao, L., Gartner, F., Vilanova, M., \& Gama, M (2010). In vivo biocompatibility and biodegradability of dextrin-based hydrogels. Journal of Bioactive and Compatible Polymers, 25(2), 141-153.

Nunes, C., Silva, L., Fernandes, A. P., Guiné, R. P. F., Domingues, M. R. M., \& Coimbra, M. A. (2012). Occurrence of cellobiose residues directly linked to galacturonic acid in pectic polysaccharides. Carbohydrate Polymers, 87(1), 620-626.

Passos, C. P., Moreira, A. S., Domingues, M. R., Evtuguin, D. V., \& Coimbra, M. A. (2014). Sequential microwave superheated water extraction of mannans from spent coffee grounds. Carbohydrate Polymers, 103(103), 333-338.

Peers, E., \& Gokal, R. (1998). Icodextrin provides long dwell peritoneal dialysis and maintenance of intraperitoneal volume. Artificial Organs, 22(1), 8-12.

Peppas, N. A., Hilt, J. Z., Khademhosseini, A., \& Langer, R. (2006). Hydrogels in biology and medicine: From molecular principles to bionanotechnology. Advanced Materials, 18(11), 1345-1360.

Schramm, C., Spitzer, M. S., Henke-Fahle, S., Steinmetz, G., Januschowski, K., Heiduschka, P., Geis-Gerstorfer, J., Biedermann, T., Bartz-Schmidt, K. U., \& Szurman, P. (2012). The cross-linked biopolymer hyaluronic acid as an artificial vitreous substitute. Investigative Ophthalmology \& Visual Science, 53(2), 613-621.

Sheu, M. T., Huang, J. C., Yeh, G. C., \& Ho, H. O. (2001). Characterization of collagen gel solutions and collagen matrices for cell culture. Biomaterials, 22(13), 17131719 .

Skehan, P., Storeng, R., Scudiero, D., Monks, A., McMahon, J., Vistica, D., Warren, J. T., Bokesch, H., Kenney, S., \& Boyd, M. R. (1990). New colorimetric cytotoxicity assay for anticancer-drug screening. Journal of the National Cancer Institute, 82(13), 1107-1112.

Su, W. Y., Chen, Y. C., \& Lin, F. H. (2010). Injectable oxidized hyaluronic acid/adipic acid dihydrazide hydrogel for nucleus pulposus regeneration. Acta Biomaterialia, 6(8), 3044-3055.

Sung, H. W., Huang, R. N., Huang, L. L., \& Tsai, C. C. (1999). In vitro evaluation of cytotoxicity of a naturally occurring cross-linking reagent for biological tissue fixation. Journal of Biomaterials Science Polymer Edition, 10(1), 63-78.

Takatori, Y., Akagi, S., Sugiyama, H., Inoue, J., Kojo, S., Morinaga, H., Nakao, K., Wada, J., \& Makino, H. (2011). Icodextrin increases technique survival rate in peritoneal dialysis patients with diabetic nephropathy by improving body fluid management: A randomized controlled trial. Clinical Journal of the American Society of Nephrology, 6(6), 1337-1344.

Tan, H. \& Hu, X. (2012). Injectable in situ forming glucose-responsive dextran-based hydrogels to deliver adipogenic factor for adipose tissue engineering. Journal of Applied Polymer Science, 126(S1), E180-E187.

Van Vlierberghe, S., Dubruel, P., \& Schacht, E. (2011). Biopolymer-based hydrogels as scaffolds for tissue engineering applications: A review. Biomacromolecules, 12(5), 1387-1408. 
von Burkersroda, F., Schedl, L., \& Gopferich, A. (2002). Why degradable polymers undergo surface erosion or bulk erosion. Biomaterials, 23(21), 4221-4231.

White, D. R., Jr., Hudson, P., \& Adamson, J. T. (2003). Dextrin characterization by highperformance anion-exchange chromatography-pulsed amperometric detection and size-exclusion chromatography-multi-angle light scattering-refractive index detection. Journal of Chromatography A, 997(1-2), 79-85.
Zhang, R., Tang, M., Bowyer, A., Eisenthal, R., \& Hubble, J. (2005). A novel pH- and ionic-strength-sensitive carboxy methyl dextran hydrogel. Biomaterials, 26(22), 4677-4683.

Zhang, Y., \& Chu, C.C. (2002). Biodegradable dextran-polylactide hydrogel networks: Their swelling, morphology and the controlled release of indomethacin. Journal of Biomedical Materials Research, 59(2), 318-328. 